\title{
Diagnóstico e estadiamento do câncer de pulmão"
}

\author{
Angelo FERnANDEZ ${ }^{1}$, FABIO B. JATENE ${ }^{2}$, MAURO ZAMBONI ${ }^{3}$
}

O câncer do pulmão pode apresentar-se sob diversas formas e vários são os meios de diagnosticá-lo. A escolha do melhor método para o seu diagnóstico depende de diferentes aspectos relacionados ao tumor, ao paciente e à habilidade da equipe médica. Dentre os métodos mais comumente utilizados para o diagnóstico do câncer do pulmão, podemos contar, além dos exames radiológicos, que não serão abordados neste artigo, a citologia do escarro, a broncofibroscopia, a punção aspirativa transtorácica e a toracoscopia, entre outros. O estadiamento reflete a extensão anatômica do câncer.

As regras de estadiamento propostas por Denoix foram adaptadas de maneira satisfatória para o câncer do pulmão e, embora tenham sofrido diversas modificações, devidas ao avanço tecnológico dos métodos de diagnóstico, são importantes para orientar o tratamento e estimar o prognóstico. Com o advento de novas modalidades de tratamento, principalmente as combinadas, o estadiamento tende a assumir importância cada vez maior, tanto na escolha da modalidade terapêutica mais adequada quanto na comparação dos resultados. (J Pneumol 2002;28(4):219-228)

\section{Diagnosis and staging of lung cancer}

Carcinoma of the lung can present in a number of guises and a number of possible and often complementary diagnostic approaches are available. The choice of procedure should reflect the presentation of the tumor, local expertise, intended management and patient preference. There are different methods of investigation: sputum cytology, fiberbronchoscopy, transbronchial biopsy, transbronchial needle aspiration, transthoracic fine needle aspiration, etc. The association of all of these methods increases the power of diagnosis. Staging is the measurement of the anatomical extent of a tumor in any given patient. The staging of cancer began with Denoix's TNM classification system, and, although several modifications were developed, it remains the basis of lung cancer staging systems up to now. Assigning patients to a particular TNM stage allows choosing the most appropriate therapy and provides prognostic information. Also, the impact of new therapeutic methods can be evaluated for efficacy and a comparison of the expected survival rates can be predicted.

Descritores - Diagnóstico. Estadiamento de neoplasias. Neoplasias pulmonares. Toracoscopia.

Key words - Diagnosis. Lung neoplasms. Neoplasm staging. Thoracoscopy.

* Trabalho realizado no Serviço de Cirurgia Torácica do Hospital das Clínicas da Faculdade de Medicina da Universidade de São Paulo (USP).

1. Médico-Assistente, Serviço de Cirurgia Torácica.

2. Chefe do Serviço de Cirurgia Torácica.

3. Pneumologista do Serviço de Tórax do INCA/MS. Diretor Científico da SBPT.

Endereço para correspondência - Angelo Fernandez, Rua da Consolação, 3.726, apto. 132 - 01416-000 - São Paulo, SP. Tel. (11) 30821256; fax (11) 3255-5999; e-mail: angelofe@usp.br

Recebido para publicação em 7/3/02. Aprovado, após revisão, em 5/6/02.
Siglas e abreviaturas utilizadas neste trabalho AJCC - American Joint Commitee on Cancer CT - Tomografia computadorizada

FDG - Fluor-Deoxi-Glicose

MRI - Ressonância magnética

PET-scan - Tomografia por emissão de positron UICC - Union Internationale Contre le Cancer

\section{INVESTIGAÇÃO DIAGNÓSTICA}

\section{CitOLOGIA DO ESCARRO}

Com a disseminação e a maior disponibilidade da broncofibroscopia, a confiabilidade na citologia do escarro vem diminuindo. Entretanto, ela é ocasionalmente útil, especialmente naqueles pacientes nos quais se necessita de uma definição diagnóstica, mas a broncofibroscopia não 
está indicada ${ }^{(1)}$. Uma única amostra de escarro é positiva em aproximadamente $40 \%$ dos pacientes portadores de câncer do pulmão. O rendimento cumulativo aumenta para $56 \%, 69 \%$ e $85 \%$, nas 2as, 3 as e 4 as amostras, respectivamente, mas depende da localização e do tamanho do tumor e os melhores resultados são obtidos quando os tumores são grandes e centrais ${ }^{(2)}$. As melhores amostras são aquelas colhidas pela manhã; mas as amostras colhidas uma a quatro horas após a broncoscopia ou na manhã seguinte da realização do exame também têm bom rendimento, mesmo o exame sendo normal ${ }^{(3,4)}$.

O rendimento da citologia do escarro é influenciado pela localização, tamanho e tipo histológico do tumor. Desse modo, os carcinomas escamosos, os quais usualmente têm localização central, geralmente representam a maioria das neoplasias pulmonares diagnosticadas pela citologia do escarro. Os adenocarcinomas, por outro lado, por ter, em grande número de casos, localização periférica, são menos diagnosticados pela análise citológica do escarro $^{(5)}$. A presença de células do adenocarcinoma na citologia do escarro, geralmente, reflete a presença de tumores grandes e inoperáveis ${ }^{(6,7)}$.

Muitos pacientes produzem pouco ou nenhum escarro $e$, nestes, outras técnicas para a coleta da amostra podem ser utilizadas ${ }^{(8,9)}$. A que apresenta melhor rendimento é a técnica da coleta do escarro após nebulização com solução salina hipertônica (escarro induzido), que tem sensibilidade de $84 \%$. A coleta do escarro através dessa técnica parece que melhora seu rendimento nos casos dos adenocarcinomas ${ }^{(10)}$.

\section{BRONCOFIBROSCOPIA}

A broncofibroscopia é, atualmente, o principal exame na avaliação diagnóstica do paciente com câncer do pulmão. Ela é útil no seu diagnóstico e também no estadiamento e no tratamento. Relativamente fácil de realizar, esse procedimento também é seguro e bem tolerado pelos pacientes. A flexibilidade do aparelho permite que o observador examine a maioria dos brônquios de quarta ordem e freqüentemente os de sexta.

As contra-indicações ao método são poucas e incluem a hipoxemia, o sangramento, a instabilidade cardiovascular e a hipercapnia.

A broncofibroscopia é um exame seguro, com índice de complicações de 0,12\% e índice de mortalidade de $0,04 \%{ }^{(11)}$.

Através da broncofibroscopia podem-se estabelecer critérios de inoperabilidade do paciente portador de câncer do pulmão. São eles: a paralisia da corda vocal, o tumor localizado a menos de $2 \mathrm{~cm}$ da carina principal e o envolvimento desta pelo tumor(12).

Embora a broncofibroscopia seja o exame endoscópico mais comumente utilizado nos pacientes com neopla- sia pulmonar, a broncoscopia rígida tem vantagens sobre ela em algumas situações. Algumas vezes, ela pode fornecer informações mais precisas sobre a localização do tumor. É capaz de avaliar melhor a mobilidade da árvore brônquica proximal, fornecendo informações indiretas sobre seu envolvimento pelos linfonodos mediastinais. Ela também está indicada nos casos de estenose da traquéia, pois permite melhor ventilação do paciente. Proporciona, também, melhor aspiração da árvore respiratória, melhor controle dos sangramentos e permite a ressecção tumoral endoscópica ${ }^{(13)}$. É o procedimento de escolha, para alguns autores, para a colocação dos suportes endobrônquicos e para a ressecção endobrônquica com laser ou eletrocautério(14).

O rendimento diagnóstico da broncofibroscopia depende da localização do tumor. Nas lesões endobrônquicas centrais têm alto rendimento: $>90 \%$.

A técnicas associadas à broncofibroscopia incluem o lavado, o escovado e a biópsia brônquica; seu rendimento pode aumentar se a elas associarmos a biópsia aspirativa transbrônquica e o lavado bronco-alveolar(15).

Mais de $70 \%$ dos carcinomas do pulmão são visíveis através da broncofibroscopia. O rendimento do exame aumenta se, a ele, associamos o lavado, o escovado e a biópsia (três a cinco fragmentos) brônquicas. Desse modo, conseguimos a definição do tipo do tumor em mais de $90 \%$ dos $\operatorname{casos}^{(16)}$. Na ausência de lesão endobrônquica visível, quando somente podemos observar comprometimento tumoral da submucosa brônquica, o rendimento do método cai para 55\% e está mais reduzido ainda quando a lesão não é vista através da broncofibroscopia(16).

Nos casos nos quais não se observam lesões endobrônquica através da broncofibroscopia, podemos lançar mão de novas técnicas com o objetivo de aumentar o rendimento do nosso exame. São elas: a biópsia transbrônquica $e$ a punção aspirativa transbrônquica por agulha ${ }^{(17)}$. A associação de todos esses métodos aumenta de maneira significativa o rendimento do exame $e^{(18)}$.

\section{BIÓPSIA TRANSBRÔNQUICA}

O rendimento diagnóstico da broncofibroscopia diminui quando as lesões estão localizadas além da vista do endoscopista. Ele é menor nas lesões periféricas e nas lesões menores do que $2 \mathrm{~cm}^{(19)}$. Nestes casos, a utilização da biópsia transbrônquica é capaz de aumentar o rendimento diagnóstico. Nas lesões localizadas e quando realizada com o auxílio da fluoroscopia, tem sensibilidade de $64 \%$. Esta sensibilidade aumenta se a ela associamos o lavado bronco-alveolar ${ }^{(19)}$. A fluoroscopia aumenta o rendimento diagnóstico da biópsia transbrônquica nas lesões focais; entretanto, é um procedimento demorado, que requer experiência e muitas vezes não disponível. Nas lesões difusas, entretanto, como na linfangite carcinomato- 
sa, o rendimento é o mesmo, caso se utilize a fluoroscopia ou não e, portanto, deve ser o método de escolha quando suspeitamos desta entidade ${ }^{(20,21)}$.

As complicações da biópsia transbrônquica incluem o pneumotórax e o sangramento, em menos de $1 \%$ dos casos.

\section{PUNÇÃO ASPIRATIVA TRANSBRÔNQUICA POR AGULHA}

A punção aspirativa transbrônquica por agulha, embora seja descrita desde 1958, e desenvolvida para ser utilizada através da broncofibroscopia desde 1983, ainda é um procedimento subutilizado, apesar do bom rendimento diagnóstico ${ }^{(22,23)}$. Ela tem sido utilizada no estadiamento dos linfonodos mediastinais, para o diagnóstico das lesões pulmonares periféricas e para aquelas endoscopicamente visíveis.

No estadiamento mediastinal, a punção aspirativa transbrônquica por agulha é utilizada para o estudo dos linfonodos localizados junto à traquéia e aos brônquios principais. Os locais da punção devem ser estabelecidos previamente com o auxílio da tomografia computadorizada do tórax. Ela deve ser o primeiro procedimento a ser realizado, durante a broncofibroscopia, com o objetivo de impedir a contaminação do material colhido com células neoplásicas exfoliadas do tumor. Nos diferentes estudos sua sensibilidade variou de 43 a 83\%(24-27).

Diferentes estudos demonstraram a utilidade da punção aspirativa transbrônquica por agulha no diagnóstico das lesões periféricas do pulmão ${ }^{(28)}$. O rendimento é maior quando o tumor está localizado na porção intermediária do pulmão do que quando situado na periferia do órgão ${ }^{(29,30)}$. Ela representa uma alternativa à biópsia transbrônquica nos casos de estenose da via aérea pelo tumor ou por compressão extrínseca. As suas complicações são semelhantes àquelas da biópsia transbrônquica.

Em algumas ocasiões, a biópsia de uma lesão endobrônquica visível não é conclusiva. Isso se deve principalmente à presença de material necrótico na superfície do tumor ou ao esmagamento celular que algumas vezes ocorre na própria coleta da amostra. Nessas circunstâncias, a punção aspirativa por agulha aumenta o rendimento diagnóstico ${ }^{(30)}$.

\section{LAVADO BRONCO-ALVEOLAR}

O principal papel do lavado bronco-alveolar nos portadores de câncer do pulmão é o diagnóstico das infecções oportunísticas nos pacientes em tratamento com quimioterapia. Entretanto, atualmente sabe-se que o lavado bronco-alveolar tem importância no diagnóstico da neoplasia pulmonar. Seu maior rendimento é encontrado nas doenças malignas hematológicas que acometem os pulmões, no carcinoma bronquíolo-alveolar e no adenocarcinoma metastático da mama para os pulmões ${ }^{(31-34)}$.
Informações sobre o rendimento do lavado bronco-alveolar no diagnóstico das neoplasias pulmonares continuam escassas. Entretanto, existem relatos de um rendimento diagnóstico de 32,7\% em tumores periféricos do pulmão. Associado aos outros métodos - lavado e escovado - o rendimento aumentou para 56\%(35).

É um procedimento seguro e suas complicações, bastante raras.

\section{PUNÇÃO-BIÓPSIA PERCUTÂNEA COM AGULHA FINA}

A biópsia percutânea por agulha foi descrita pela primeira vez em 1883 como um método para o diagnóstico das pneumonias ${ }^{(36)}$ e somente em 1886 foi utilizada para estabelecer o diagnóstico de um tumor do pulmão(37). As complicações do método, especialmente o pneumotórax, nessa ocasião, impediram a sua disseminação. Somente muitos anos mais tarde, com o desenvolvimento de agulhas de paredes finas, do intensificador de imagens e das técnicas citológicas, esse método diagnóstico ressurgiu e hoje é considerado o procedimento de escolha para o diagnóstico dos tumores periféricos dos pulmões. O exame é realizado com o auxílio da fluoroscopia, da ultrasonografia ou da tomografia computadorizada, sob anestesia local e com praticamente nenhum desconforto para o paciente $e^{(38)}$. Sua sensibilidade varia de 70 a $100 \%$ e depende da localização e do tamanho da lesão, sendo mais intensa nos tumores maiores do que $3 \mathrm{~cm}^{(39)}$. O pneumotórax é a maior complicação do exame, mas somente um pequeno número de casos necessita de drenagem torácica. Menos comuns são os sangramentos pós-punção.

\section{DiAgNóSTICO DA DOENÇA PLEURAL}

O derrame pleural é uma manifestação comum dos tumores primários e metastáticos do pulmão e da pleura. $\mathrm{O}$ rendimento diagnóstico da citologia do líquido pleural varia de 50 a $75 \%$ dos casos $^{(40)}$. A toracocentese deve ser sempre realizada concomitantemente com a biópsia pleural com agulha. O rendimento da biópsia pleural varia de 40 a $70 \%(41)$. Quando associamos os dois métodos, o rendimento aumenta significativamente e pode alcançar 8090\%(41).

Quando utilizamos a ultra-sonografia ou a tomografia computadorizada para orientar a biópsia pleural seu rendimento aumenta, pois se pode ter acesso aos locais mais comprometidos da pleura ${ }^{(42,43)}$.

As complicações da biópsia pleural são raras. O pneumotórax é o mais comum e, geralmente, é pequeno e assintomático e tem resolução espontânea ${ }^{(44)}$.

Significativo número de derrames pleurais permanece sem diagnóstico, mesmo após a toracocentese e a biópsia pleural. Nesses casos, a toracoscopia está indicada. A inspeção direta do espaço pleural permite ao examinador identificar o melhor local para a biópsia. Como espe- 
rado, a toracoscopia aumenta o rendimento diagnóstico e resultados de até 95\% de positividade já foram relatados na literatura(45). A toracoscopia é uma técnica invasiva, mas com baixos índices de mortalidade $(<0,01 \%)^{(46)}$. Poucas são as contra-indicações relativas: diátese hemorrágica, hipoxemia, instabilidade cardiovascular e tosse incontrolável.

\section{DiagnÓstico DA DOENÇA MEDIASTINAL}

A mediastinoscopia facilita o estadiamento patológico dos linfonodos mediastinais( ${ }^{(47)}$. Através de uma incisão cervical, dissecando-se a fáscia anterior da traquéia consegue-se biopsiar os linfonodos paratraqueais altos e baixos, pré-traqueais e, em alguns casos, os hilares e carinais. $\mathrm{O}$ acesso aos linfonodos subcarinais e aos da janela aorto-pulmonar somente é conseguido através da mediastinostomia anterior. A mediastinoscopia tem rendimento diagnóstico que varia de 89 a $100 \%{ }^{(48)}$. Ambos os procedimentos são realizados sob anestesia geral com mínima morbidade e mortalidade: respectivamente, 0,6\% e $0,2 \%(49)$. A complicação mais comum é o sangramento, mas somente um número insignificativo de casos necessita de toracotomia para contê-lo. Outras complicações menos comuns são: paralisia da corda vocal, infecção da ferida operatória, mediastinite, perfuração do esôfago, laceração da artéria pulmonar e broncoespasmo.

A mediastinoscopia cervical oferece acesso limitado aos linfonodos da janela aorto-pulmonar, do mediastino anterior e da região subcarinal. Nestes casos, a videotoracoscopia pode ser uma alternativa à mediastinotomia anterior.

\section{ESTADIAMENTO}

\section{CONSIDERAÇÕES INICIAIS}

O principal objetivo dos esquemas de classificação, que foram chamados genericamente de métodos de estadiamento, é estabelecer um modo de determinar a extensão anatômica das neoplasias, permitindo uniformização do tratamento e a comparação dos resultados. Para que estes esquemas de classificação fossem aceitos, foram atrelados a critérios uniformes e a regras que poderiam ser alteradas em função de métodos diagnósticos mais sofisticados ou novas terapêuticas que fossem surgindo ${ }^{(50,51)}$.

Denoix $^{(52)}$, em 1946, idealizou o sistema que seria conhecido como sistema TNM. Ele se baseia em critérios lógicos simples, adaptáveis a quase todos os tumores sólidos. Os critérios de classificação estão ligados ao tamanho e posição do tumor primário (T), à presença e localização de linfonodos comprometidos $(\mathrm{N})$ e à presença de metástases a distância. A cada um desses parâmetros são dados subscritos, que permitem estimar a extensão ana- tômica da doença e, a partir daí, associar estes dados ao tratamento e à sobrevida ${ }^{(53)}$.

\section{O SISTEMA TNM E AS NEOPLASIAS PULMONARES}

O câncer do pulmão não é uma doença de comportamento uniforme, pois engloba diversos tipos histológicos, com atividade biológica e agressividade diferentes. Conhecer o tipo histológico e a extensão anatômica da doença é fundamental para indicar o tratamento adequado e prever o prognóstico de cada caso ${ }^{(54)}$.

$\mathrm{O}$ estadiamento do câncer do pulmão baseia-se nos critérios anatômicos do sistema TNM. O estadiamento não inclui outros fatores prognósticos como o performance status, a condição cardiorrespiratória, idade, tipo histológico e invasão extracapsular. $\mathrm{O}$ estadiamento reflete a ressecabilidade, ou seja, a possibilidade de remover cirurgicamente toda a neoplasia. Por outro lado, a operabilidade é dada pelo estadiamento e por todos os outros fatores acima mencionados(55).

O sistema TNM foi adaptado às neoplasias pulmonares não pequenas células no fim da década de 60. Desde então, passou por várias modificações, adequando-se a métodos diagnósticos mais eficientes e a terapêutica mais avançada. A popularização da tomografia computadorizada a partir da década de 80 fez com que os métodos de imagem assumissem importância fundamental no estadiamento.

Dados publicados nos anos 80 mostraram a necessidade de conhecer com precisão a extensão da doença antes de indicar o tratamento. Ao mesmo tempo em que as operações mais extensas eram sistematizadas, percebiase a importância das metástases linfonodais como fatores limitantes da sobrevida e a mediastinoscopia começa a ter um papel importante no estadiamento. A partir daí, as modificações instituídas nos esquemas de classificação superam as deficiências dos métodos mais antigos, determinando um sistema mais simples, objetivo e preciso, compreendido e aceito por todos os elementos da equipe multidisciplinar envolvida no tratamento do câncer pulmonar $^{(56)}$.

Os esquemas de estadiamento modernos exigem precisão, pois tendem a orientar a indicação cirúrgica e o tratamento neo-adjuvante e adjuvante. As regras atuais distinguem o tumor com invasão local potencialmente ressecável (T3) da invasão não ressecável (T4). Da mesma forma, as metástases para linfonodos mediastinais cirurgicamente ressecáveis, que são caracterizados como N2, são diferenciadas dos linfonodos mediastinais contralaterais ou extratorácicos (supraclaviculares, pré-escalênicos, cervicais), agora denominados N3. Estas lesões, embora consideradas não cirúrgicas, são acessíveis à terapêutica localizada (radioterapia). 
Em 1997, estudando os grupos cooperativos dos últimos 10 anos, Mountain publicou a mais recente revisão dos critérios de estadiamento aceitos pela American Joint Commitee on Cancer (AJCC) e Union Internationale Contre le Cancer (UICC) ${ }^{(57)}$. Os agrupamentos foram reorganizados e novas classificações foram abertas. Assim, os grupos I e II, que acolhem a doença localizada, têm indicação de tratamento semelhante (cirurgia) e foram subdivididos para acolher pacientes cujo prognóstico é mais próximo. O grupo T3NOM0 foi deslocado para o estádio IIB, pois seu prognóstico é melhor que a classificação anterior, que o colocava como IIIA. A divisão do estádio III em dois subgrupos segue a mesma orientação: a divisão IIIA caracteriza pacientes com prognóstico reservado, que apresentam doença ressecável, e o estádio IIIb representa o grupo de pacientes com doença irressecável. Finalmente, os pacientes não enquadrados no estádio IV são aqueles que necessitam de tratamento sistêmico por apresentar doença disseminada. Nódulos satélites intraparenquimatosos foram classificados como T4. Nódulos em outros lobos foram classificados como M1. As regras atuais utilizadas para o estadiamento do câncer de pulmão são as seguintes:

\section{Tumor primário $(\mathrm{T})$}

Tx...........Tumor provado pela presença de células neoplásicas nas secreções broncopulmonares ou em lavados broncoalveolares, porém não identificado pela radiografia ou broncoscopia, ou qualquer tumor que não possa ser localizado.

T0........... Nenhuma evidência de tumor primário.

T1s..........Carcinoma in situ.

T-1.........Tumor com menos de $3 \mathrm{~cm}$ no seu maior diâmetro, circundado por pleura ou tecido pulmonar em toda a sua extensão, sem evidência de invasão proximal a um brônquio lobar ao exame endoscópico.

T-2 .........Tumor com qualquer das seguintes características:

Mais de $3 \mathrm{~cm}$ no maior diâmetro;

Invade a pleura visceral;

Provoca atelectasia ou pneumonite crônica obstrutiva;

Estende-se à região hilar, porém, à broncoscopia, o tumor deve estar a pelo menos $2 \mathrm{~cm}$ distal à carina principal.

T-3..........Tumor de qualquer dimensão, com:

Invasão direta da parede torácica (incluindo tumores do sulco superior), do diafragma, do pericárdio ou pleura mediastinal, sem invadir estruturas viscerais, grandes vasos ou corpo vertebral;

Tumores de qualquer tamanho localizados a menos de $2 \mathrm{~cm}$ da carina principal.

T-4 .........Tumor de qualquer tamanho invadindo mediastino, comprometendo coração, grandes vasos, tra- quéia, esôfago, corpo vertebral ou carina, ou tumores de qualquer dimensão, acompanhados de derrame pleural com citologia positiva.

\section{Linfonodos (N)}

Nx..........Metástases linfonodais não demonstráveis.

N-0..........Ausência de metástases linfonodais.

$\mathbf{N}-1$...........Metástases linfonodais para a região peribrônquica, linfonodos hilares ipsilaterais ou ambos, incluindo extensão direta (cadeias 10, 11).

$\mathbf{N}-2$..........Metástases para linfonodos mediastinais ipsilaterais ou subcarinais (cadeias 2, 4, 5, 6, 7, 8, 9 homolaterais).

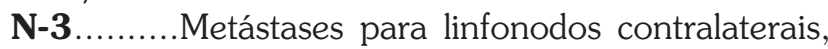
mediastinais ou hilares ou linfonodos cervicais ou préescalênicos, ipsi ou contralaterais.

\section{Metástases a distância (M)}

M x...........Metástases não demonstradas.

M-0..........Ausência de metástases.

M-1 ..........Presença de metástases a distância.

\section{ESTADIAMENTO FINAL}

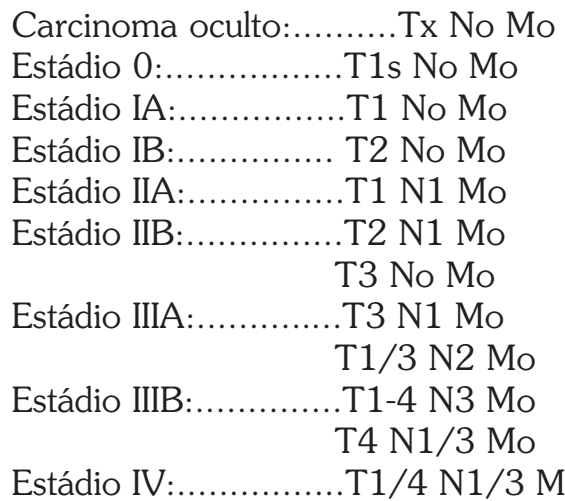

$\mathrm{O}$ estadiamento moderno envolve avaliações complexas que incluem clínico, cirurgião, endoscopista, radiologista e patologista. A importância dos métodos de imagem é cada vez mais evidente, principalmente no que concerne à avaliação dos critérios de ressecabilidade.

\section{Estadiamento intratorácico}

O estadiamento intratorácico determina a ressecabilidade, através dos parâmetros T e N. Para esta avaliação contribuem a tomografia computadorizada e os métodos cirúrgicos de estadiamento linfonodal, ou sejam, a mediastinoscopia e a videotoracoscopia. Recentemente, a ressonância nuclear magnética foi usada como método para estadiamento, tanto sistêmico como intratorácico. Infelizmente, seus resultados não foram superiores aos mostrados pela tomografia computadorizada, exceto na avaliação dos tumores do sulco superior que apresentavam a síndrome de Pancoast e na investigação da inva- 
são de grandes vasos. O PET-scan (positron emission tomography) mostra uma perspectiva muito favorável para o estadiamento linfonodal e sistêmico, mas ainda não há experiência significativa e os resultados não são conclusivos.

\section{Avaliação do T}

O parâmetro T é importante quando se pensa em ressecção cirúrgica. As regras de estadiamento atual dividem o tumor primário em quatro grupos (T1 a T4), considerando as classificações T1, T2 e T3 como formas ressecáveis e definindo como T4 a lesão habitualmente não ressecável. A tomografia computadorizada aliada à broncoscopia é usada para diferenciar as lesões T3 das T4. Apesar do seu alto poder de resolução, o exame é falho em mais de 30\% dos casos de diagnóstico diferencial entre T3 e T4, já que as imagens muitas vezes não conseguem caracterizar invasão de estruturas mediastinais quando estão muito próximas ao tumor. Da mesma forma, a ausência de planos de clivagem identificáveis nas imagens também não é parâmetro fidedigno de invasão. Em casos selecionados, a ressonância nuclear magnética melhorou a avaliação de invasão da parede, principalmente nos tumores do sulco superior e nas suspeitas de invasão de grandes vasos.

\section{Avaliação do N}

O acometimento linfático é um dos fatores prognósticos mais importantes no câncer do pulmão, sendo considerado o principal divisor de águas entre o tratamento cirúrgico e o não cirúrgico nos portadores de doença exclusivamente intratorácica.

A fisiologia do fluxo linfático mediastinal e a forma com que as células neoplásicas são transportadas na linfa e as suas implantações nos linfonodos foram muito estudadas. Não existe um padrão constante no fluxo linfático, embora a tendência normal seja um fluxo direcionado de forma centrípeta e ascendente. Da mesma forma, a tendência dos linfonodos é de reter as células neoplásicas, porém eventualmente as células podem passar por diversas estações antes de ser apreendidas pelo sistema reticuloendotelial do linfonodo. Isso dá origem às chamadas skipping metastasis, ou sejam, metástases que ultrapassaram várias estações linfáticas antes de se fixar em determinado linfonodo. Existe atualmente uma corrente que valoriza o linfonodo sentinela. Algumas técnicas usando radioisótopos são recomendados para localizar esse linfonodo, mas esta prática ainda não se mostrou efetiva e é objeto de estudo de diversos grupos(58).

A posição dos linfonodos comprometidos é importante e tem valores prognósticos diferentes ${ }^{(59)}$. A definição antiga de linfonodos hilares e linfonodos mediastinais é imprópria, pois não tem conotação anatômica precisa. As estações linfonodais são baseadas nos achados da to- mografia computadorizada e mediastinoscopia, que caracterizam com precisão a posição dos linfonodos e seus limites são dados por estruturas anatômicas de fácil identificação. Para superar dificuldades idealizou-se um mapa simplificado do mediastino capaz de atender às necessidades práticas e convencionou-se que o estadiamento linfático intratorácico deve seguir os seguintes princípios para assegurar um diagnóstico clínico (pré-toracotomia) mais acurado possível(60):

1) As estações linfonodais devem evitar o uso dos termos "hilar" e "mediastinal", já que estes carecem de especificidade anatômica.

2) As estações linfonodais identificadas na tomografia computadorizada podem ser transpostas esquematicamente para um mapa do mediastino com referências anatômicas identificáveis.

3) O mapa deve ser aceito por toda a equipe médica; os dados clínicos e cirúrgicos obtidos durante a investigação e tratamento deveriam ser transpostos passo a passo.

Os mapas usados para o estadiamento linfático estão mostrados nas Figuras 1 e 2 . As cadeias 10 e 11 são consideradas como $\mathrm{N} 1 \mathrm{e}$ as cadeias 2, 4, 5, 6, 7, 8 e 9, como N2 quando homolaterais ao tumor primário. Qualquer cadeia comprometida contralateral ao tumor primário ou de localização extratorácica determina o estadiamento N3.

As metástases para linfonodos mediastinais no câncer do pulmão podem assumir diversas formas, de acordo com o poder de invasão, agressividade e tempo de evolução da doença. O estádio N2 ou N3 pode caracterizar-se por diferentes graus de acometimento mediastinal ${ }^{(61)}$, que podem ser classificados em cinco níveis:

Clínico: quando existem indícios clínicos do comprometimento, manifestados, por exemplo, pela síndrome de cava superior ou pela paralisia de corda vocal. O quadro radiológico desses pacientes é evidente e, normalmente, não traz dúvidas de interpretação.

Radiológico: quando existem linfonodos mediastinais aumentados identificados ao exame radiológico. Este critério é falho, já que é um critério morfológico sem comprovação anatomopatológica. A dificuldade é determinar qual o tamanho normal de um linfonodo não comprometido.

Mediastinoscópico: quando o comprometimento é identificado através da mediastinoscopia, sem suspeita clínica ou radiológica prévia.

Cirúrgico: quando o achado de linfonodos comprometidos só se dá durante a operação, já que tais lesões não foram investigadas ou não foram identificadas pelos métodos anteriores.

Anatomopatológico: quando pequenos linfonodos comprometidos são identificados nos cortes seriados da gordura mediastinal da peça cirúrgica ressecada. 


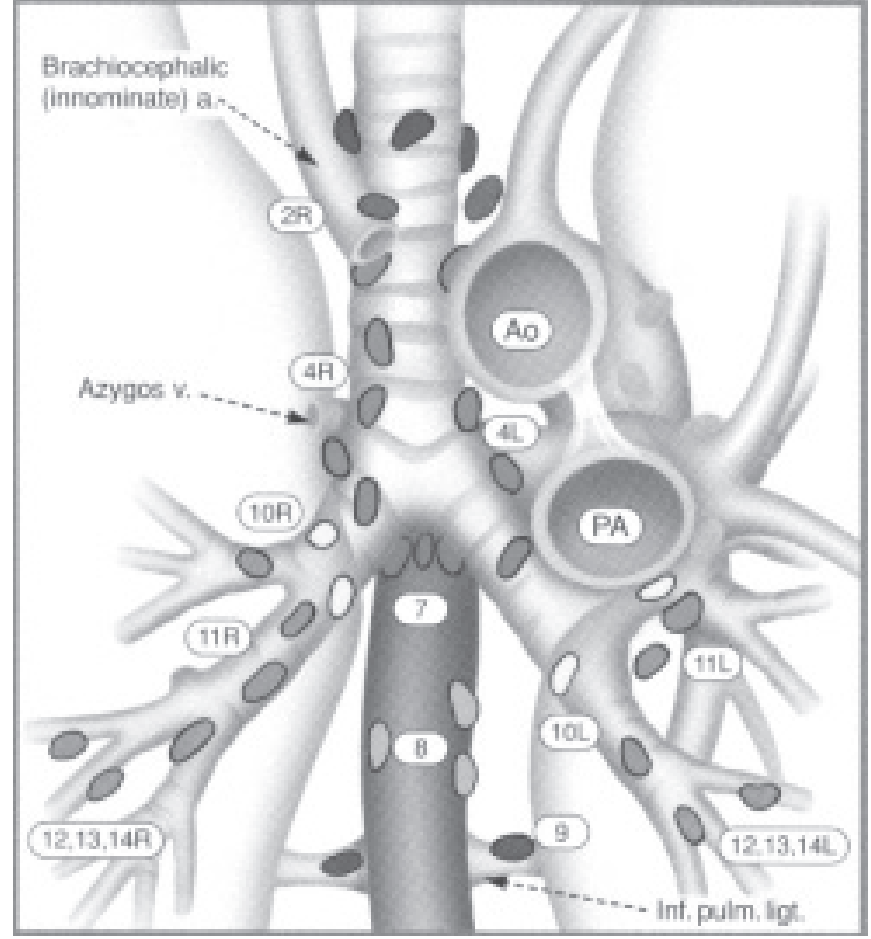

Figura 1 - Estações nodais regionais para estadiamento do câncer do pulmão. Visão frontal.

Para uma análise crítica adequada é fundamental conhecer a acurácia da tomografia computadorizada ${ }^{(62)}$. Os aparelhos de tomografia helicoidal mais recentes geram imagens precisas do mediastino, permitindo identificar e medir linfonodos em todas as estações linfáticas mediastinais. A tendência internacional considera que linfonodos com diâmetros transversos maiores que $20 \mathrm{~mm}$ têm grandes possibilidades de ser linfonodos neoplásicos. Infelizmente, o tamanho não é um critério seguro para considerar um linfonodo como positivo, principalmente em nosso meio. Linfonodos aumentados podem ser secundários a doenças inflamatórias e corresponder a falsopositivos. Da mesma forma, linfonodos de tamanho normal podem estar comprometidos, correspondendo a falso-negativos. A Tabela 1 mostra a relação entre o tamanho e o comprometimento neoplásico de 638 linfonodos

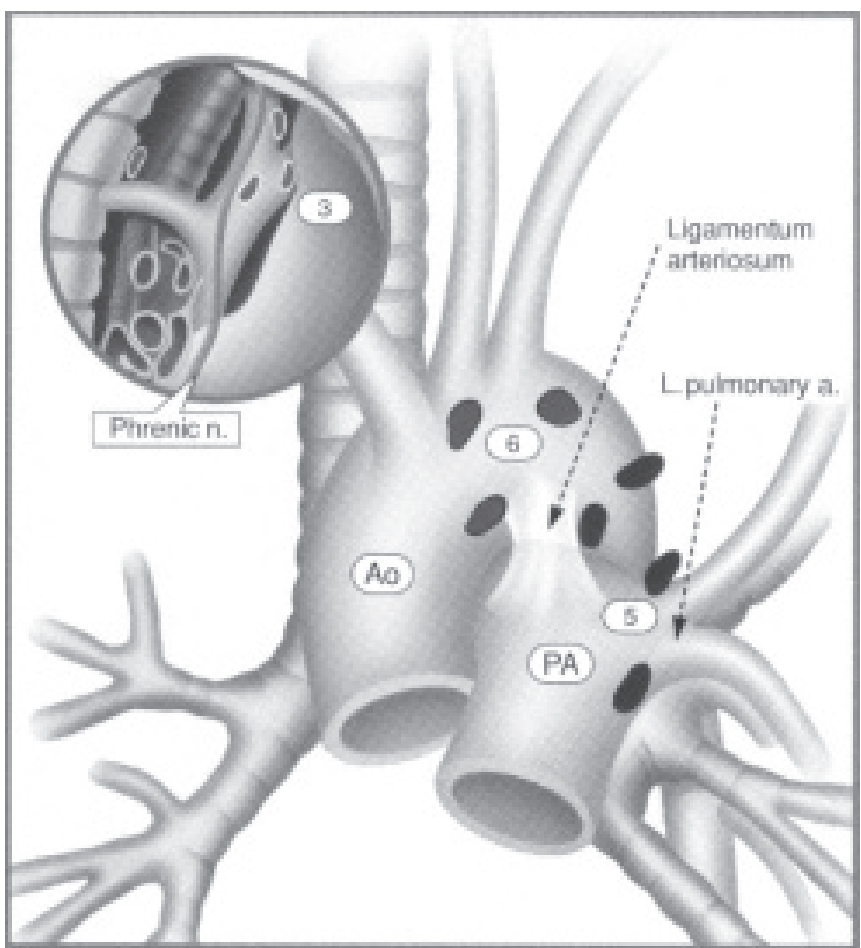

Figura 2 - Estações nodais regionais para estadiamento do câncer do pulmão. Visão da janela aorto-pulmonar.

ressecados em 50 operações para tratamento do câncer de pulmão em nosso meio.

A Tabela 1 mostra que se aceitarmos $20 \mathrm{~mm}$ como tamanho limite para considerar um linfonodo como sendo positivo, teremos mais de $8 \%$ de falso-negativos. Da mesma forma, se mantivermos esse mesmo limite, teremos mais de $60 \%$ de falso-positivos. É por esse motivo que preconizamos a biópsia dos linfonodos suspeitos sempre que o diagnóstico de acometimento mediastinal for importante para a definição da terapêutica ${ }^{(63)}$.

\section{O papel da mediastinoscopia}

A mediastinoscopia cervical possibilita a biópsia de linfonodos das cadeias 2R, 2L, 4R, 4L e 10R (Figuras 1 e 2). As cadeias $10 \mathrm{~L}$ e 7 só podem ser atingidas se os linfonodos são muito grandes. As cadeias pré-aórticas (5 e 6)

TABELA 1

Relação entre tamanho e comprometimento neoplásico de linfonodos

\begin{tabular}{lccc}
\hline \multicolumn{1}{c}{ Tamanho } & No linfonodos & Comprometidos & \% comprometimento \\
\hline$<10 \mathrm{~mm}$ & 329 & 10 & $3,03 \%$ \\
$>10 \mathrm{e}<20 \mathrm{~mm}$ & 211 & 11 & $5,21 \%$ \\
$>20 \mathrm{e}<30 \mathrm{~mm}$ & 52 & 12 & $23,07 \%$ \\
$>30 \mathrm{~mm}$ & 46 & 12 & $26,08 \%$ \\
\hline
\end{tabular}


e mediastinais posteriores (8 e 9) são inacessíveis ${ }^{(64)}$. Como podemos observar, a mediastinoscopia acessa o espaço "retrocava e pré-traqueal", que é o espaço para onde drena a maior parte do fluxo linfático do tórax.

A sensibilidade da CT e mediastinoscopia é semelhante (64\% para a tomografia e 58\% para a mediastinoscopia). Por outro lado, a especificidade da mediastinoscopia é de $100 \%$, já que permite estudo histopatológico, enquanto a especificidade da tomografia é de $63 \%(65)$, pois avalia exclusivamente fenômenos morfológicos. Estes dados nos permitem concluir que os métodos são complementares ao adequado estadiamento linfático na fase pré-toracotomia.

Como a mediastinoscopia investiga as cadeias linfáticas mediastinais altas e paratraqueais, outras técnicas foram propostas para alcançar as cadeias pré-aórticas (mediastinoscopia anterior, hiloscopia, mediastinoscopia ampliada) ou as cadeias posteriores (mediastinoscopia posterior ou pleuromediastinoscopia). Com a popularização da videotoracoscopia, o acesso a todas as cadeias linfáticas intratorácicas pode ser conseguido na fase pré-toracotomia imediata, com baixo risco, alta sensibilidade $e$ especificidade e com trauma mínimo ${ }^{(66,67)}$.

Com a popularização do tratamento combinado, ou seja, neo-adjuvante nos estádios IIIA e IIIB, conhecer o estadiamento mediastinal é fundamental para a indicação da terapêutica. A biópsia de linfonodos mediastinais passa a ser fundamental quando há linfonodos aumentados.

\section{METÁSTASES A DISTÂNCIA}

A pesquisa de metástases extratorácicas é importante, tanto para a escolha do tratamento sistêmico adequado, quanto para evitar uma intervenção cirúrgica desnecessária, já que, regra geral, não há indicação cirúrgica nos portadores de doença metastática, exceção feita nos raros casos de metástase única(68).

A história clínica e exame físico rigoroso, por si, já podem induzir a suspeitas diagnósticas, dirigindo os exames subsidiários. Perda de peso, astenia importante, dores ósseas localizadas, alterações funcionais ou metabólicas, cefaléia ou alterações do exame neurológico devem ser pesquisadas através dos exames pertinentes. Alterações de exames laboratoriais (enzimas hepáticas, cálcio, DHL) também aumentam as suspeitas de lesões a distância ${ }^{(69)}$.

O estadiamento clássico para os portadores de câncer de pulmão inclui, além da tomografia do tórax, a tomografia de crânio (indica-se ressonância quando o paciente não pode usar contraste ou quando há lesões duvidosas na CT), tomografia ou ultra-sonografia do abdome superior e mapeamento ósseo.

Há muita controvérsia sobre a real necessidade de promover o estadiamento completo e sistemático em todos os pacientes com neoplasia pulmonar ${ }^{(70)}$. A relação cus- to-benefício tem um peso muito importante nessa equação. A tendência atual é de que o estadiamento sistemático e completo seja indicado seletivamente. Estudos para avaliar a importância do estadiamento completo mostraram que a pesquisa sistemática nos estádios I e II, quando não há linfonodos mediastinais comprometidos, tem baixa positividade, principalmente no carcinoma epidermóide. As indicações mais comuns para o estadiamento completo em pacientes absolutamente assintomáticos e com diagnóstico de neoplasia pulmonar confirmada são:

Presença de adenopatia mediastinal.

Pacientes IIB ou IIIA, com indicação cirúrgica.

Pacientes com doença estádio I ou IIA, mas com alto risco cirúrgico.

Pacientes com história de perda de peso recente.

\section{Metástases no sistema nervoso central}

A incidência de metástases cerebrais em pacientes que morrem de carcinoma pulmonar varia de $30 \%$ a $50 \%$. De todos os métodos de diagnósticos para detecção de metástase cerebral, o mais utilizado é a tomografia computadorizada.

Com o advento da ressonância magnética (MRI), aumentou a sensibilidade para identificar lesões metastáticas menores que $1 \mathrm{~cm}$. A ressonância é indicada em situações em que a tomografia deixa dúvidas ou quando é normal, mas o exame neurológico está alterado. As imagens em T2 de MRI são sensíveis para localizar o edema que acompanha a maioria das metástases cerebrais. A desvantagem é o custo, que impede sua utilização como primeiro método de avaliação.

\section{Metástases supra-renais}

Relatos de necropsia mostram que de $35-38 \%$ dos pacientes falecidos com câncer de pulmão têm metástases supra-renais. Com a indicação mais freqüente da tomografia ou ultra-sonografia de abdome, massas adrenais foram encontradas em cerca de $10 \%$ dos pacientes com câncer pulmonar. Como as lesões benignas das suprarenais são entidades freqüentes (projeta-se que de 1 a $8 \%$ da população acima de 60 anos seja portadora de crescimentos benignos da supra-renal), há necessidade de biópsia dirigida quando nelas encontramos lesões expansivas. A ressonância magnética das supra-renais não se mostrou superior à tomografia.

\section{METÁSTASES HEPÁTICAS}

Metástases hepáticas geralmente são assintomáticas. Elevação das enzimas (TGO, TGP, fosfatase alcalina ou DHL) são suspeitas e devem ser investigadas. Ultra-sonografia, tomografia ou ressonância são usados na investigação de possíveis metástases hepáticas. Punções-biópsias guiadas, laparoscopia ou, mais raramente, laparotomia podem ser necessárias para confirmar uma imagem suspeita. 


\section{Metástases ósseas}

A disseminação óssea do câncer de pulmão é relativamente freqüente, principalmente no adenocarcinoma avançado. O mapeamento ósseo com Tc-99m ainda é o exame mais sensível para pesquisa de metástase óssea. Infelizmente, por ser pouco específico, tem alto índice de falso-positivos. No caso de dúvida diagnóstica, deve-se recorrer a investigações adicionais, indicando radiografia, tomografia ou mesmo ressonância dos focos suspeitos. A biópsia óssea pode ser necessária em situações especiais. No carcinoma de pequenas células, a medula óssea é um local comum de metástase, podendo estar associada à presença de anemia leve ou trombocitopenia. Nos portadores de carcinoma de pequenas células, a biópsia de medula óssea está sempre indicada, pois há grande incidência de invasão nestas situações.

\section{O PET-scan (tomografia por emissão de positron) no estadiamento do câncer do pulmão}

A idéia de fazer estadiamento de todo paciente com câncer de pulmão com um só exame parece promissor. Estudos recentes referem boas perspectivas usando o PETscan e a fluor-deoxi-glicose (FDG) como marcador. O exame parece promissor na investigação de nódulos pulmonares, de metástases mediastinais e na identificação de lesões em outros órgãos. PET-scans de corpo inteiro já mostraram metástases ocultas, não detectadas em outros métodos de imagem ${ }^{71,72)}$. Como esse método ainda é muito recente e apresenta custo muito alto, além de não ser disponível em muitos centros, é prudente aguardar sua popularização e estudos mais precisos sobre sua acurácia no estadiamento do câncer ${ }^{(73)}$.

\section{REFERÊNCIAS}

1. Gledhill A, Bates C, Henderson D, DaCosta P, Thomas G. Sputum cytology: a limited role. J Clin Pathol 1997;50:566-8.

2. Oswald NC, Hinson KF, Canti G, Miller AB. The diagnosis of primary lung cancer with special reference to sputum cytology. Thorax 1971; 26:623-7.

3. Ng AB, Horak GC. Factors significant in the diagnostic accuracy of lung cytology in bronchial washing and sputum samples. Acta Cytol 1983;27:397-402.

4. Funahashi A, Browne TK, Houser WC, Hranicka LJ. Diagnostic value of bronchial aspirate and postbronchoscopic sputum in fiberoptic bronchoscopy. Chest 1979;76:514-7.

5. Frost JK, Ball WC, Levin ML, Tockman MS, Baker RR, Carter D, et al. Early lung cancer detection: results of the initial (prevalence) radiologic and cytologic screening in The Johns Hopkins Study. Am Rev Respir Dis $1984 ; 130: 549-54$.

6. Miura H, Konaka C, Kawate N, Tsuchida T, Kato H. Sputum cytologypositive, bronchoscopically negative adenocarcinoma of the lung. Chest 1992;102:1328-32.

7. Fontana RS, Sanderson DR, Taylor WF. Early lung cancer detection: summary and conclusions. Am Rev Resp Dis 1984;130:565-70.
8. Pedersen B, Brons M, Holm K, Pedersen D, Lund C. The value of provoked expectoration in obtaining sputum samples for cytologic investigation. A prospective, consecutive and controlled investigation of 134 patients. Acta Cytol 1985;29:750-2.

9. Khajotia RR, Mohn A, Pokieser L, Schalleschak J, Vetter N. Induced sputum and cytological diagnosis of lung cancer. Lancet 1991;338: 976-7.

10. Jack CI, Sheard JD, Lippitt B, Fromholtz A, Evans CC, Hind CR. Lung cancer in elderly patients: the role of induced sputum production to obtain a cytological diagnosis. Age Ageing 1993;22:227-9.

11. Simpson FG, Arnold AG, Purvis A, Belfield PW, Muers MF, Cooke NJ. Postal survey of bronchoscopic practice by physicians in United Kingdom. Thorax 1986;41:311-7.

12. Goldstraw P. Principles of thoracic surgery. In: Brewis RAL, Gibson GJ, Geddes DM, editors. Respiratory medicine. London: Balliere Tindall, 1990;349-69.

13. Hanson P, Collins J. In: Brewis RAL, Gibson GJ, Geddes DM, editors. Respiratory medicine. London: Balliere Tindall, 1990;317-29.

14. George PJ, Garret CP, Nixon C, Hetzel MR, Nanson EM, Millard FJ. Laser treatment for tracheobronchial tumors: local or general anaesthesia? Thorax 1987;42:656-60.

15. Mak VH, Johnston ID, Hetzel MR, Grubb C. Value of washings and brushings at fibreoptic bronchoscopy in the diagnosis of lung cancer. Thorax 1990;45:373-6.

16. Gellert AR, Rudd RM, Sinh G, Geddes DM. Fibreoptic bronchoscopy: effect of experience of operator on diagnostic yield of bronchial biopsy in bronchial carcinoma. Br J Dis Chest 1982;76:397-9.

17. Cicconetti F, Teodori L, Persiani M, DiTondo U, Alo P, Marci A, et al. Increased number of cancer cells in bronchial washing fluid detected by combining conventional cytology and high flow cytometry. J Cancer Res Clin Oncol 1997;123:571-7.

18. Shure D, Fedullo PR. Transbronchial needle aspiration in the staging of peripheral masses. Am Rev Respir Dis 1983;128:1090-2.

19. Shiner RJ, Rosenman J, Katz I, Reichart N, Hershko E, Yellin A. Bronchoscopic evaluation of peripheral lung tumors. Thorax 1988;43:8879.

20. Anders GT, Johnson JE, Bush BA, Matthews JI. Transbronchial biopsy without fluoroscopy: a seven-year perspective. Chest 1988;94:55760.

21. Aranda C, Sidhu G, Sasso LA, Adams FV. Transbronchial lung biopsy in the diagnosis of lymphangitic carcinomatosis. Cancer 1978;42:19958.

22. Schieppta E. Mediastinal lymph node puncture through the tracheal carina. Surg Gynaecol Obstet 1958;110:243-6.

23. Wang KP, Terry P. Transbronchial needle aspiration in the diagnosis and staging of bronchogenic carcinoma. Am Rev Respir Dis 1983;127: 344-7.

24. Wang KP, Brower R, Haponik EF, Siegelman S. Flexible transbronchial needle aspiration for staging of bronchogenic carcinoma. Chest 1983; 84:571-6.

25. Schenk DA, Bower JH, Bryan CL, Currie RB, Spence TH, Duncan $\mathrm{CA}$, et al. Transbronchial needle aspiration staging of bronchogenic carcinoma. Am Rev Respir Dis 1986;134:146-8.

26. Schenk DA, Strollo PJ, Pickard JS, Santiago RM, Weber CA, Jackson $\mathrm{CB}$, et al. Utility of the Wang 18 gauge transbronchial histology needle in the staging of bronchogenic carcinoma. Chest 1989;96:272-4.

27. Schenk DA, Chambers SL, Derdak S, Komadina KH, Pickard JS, Strollo $\mathrm{PJ}$, et al. Comparison of the Wang 19 and 22 gauge needles in the mediastinal staging lung cancer. Am Rev Respir Dis 1993;147:12518.

28. Chechani V. Bronchoscopic diagnosis of solitary pulmonary nodules and lung masses in the absence of endobronchial abnormality. Chest 1996;109:620-5. 
29. Reichenberger F, Weber J, Tamm M, Bolliger CT, Dalquen P, Perruchoud AP, et al. The value of transbronchial needle aspiration in the diagnosis of peripheral pulmonary lesions. Chest 1999; 116:704-8.

30. Jones DF, Chin R, Cappellari JO, Haponik FF. Endobronchial needle aspiration in the diagnosis of small cell lung carcinoma. Chest 1994; 105:1151-4

31. Morales FM, Matthews JL. Diagnosis of parenchymal Hodgkin's disease using bronchoalveolar lavage. Chest 1987;91:785-7.

32. David WB, Gadek JE. Detection of pulmonary lymphoma by bronchoalveolar lavage. Chest 1987;91:787-90.

33. Radio SJ, Rennard SI, Kessinger A, Vaughan WP, Linder J. Breast carcinoma in bronchoalveolar lavage: a cytologic and immunocytochemical study. Arch Pathol Lab Med 1989;113:333-6.

34. Springmeyer SC, Hackman R, Carlson JJ, McClellan JE. Bronchioloalveolar cell carcinoma diagnosed by bronchoalveolar lavage. Chest 1983;83:278-9.

35. De Gracia J, Bravo C, Miravitlles M, Tallada N, Orriols R, Bellmunt J, et al. Diagnostic value of bronchoalveolar lavage in peripheral lung cancer. Am Rev Respir Dis 1993;147:649-52.

36. Leyden H. Uber infectiose pneumonie. Dtsch Med Wochenschr 1883; 9:52-4.

37. Menetrier P. Cancer primitif du poumon. Bull Soc Anat du Paris 1886; 11:643.

38. Goldberg-Khahn B, Healy JC, Bishop JW. The cost of diagnosis: a comparison of four different strategies in the workup of solitary radiographic lung lesions. Chest 1997;111:870-6.

39. Westcott JL, Rao N, Colley DP. Transthoracic needle biopsy of small pulmonary nodules. Radiology 1997;202:97-103

40. Dines DE, Pierre RV, Franzen SJ. The value of cells in the pleural fluid in the differential diagnosis. Mayo Clin Proc 1975;50:571-2.

41. Von Hoff DD, Li Volsi V. Diagnostic reliability of needle biopsy of the parietal pleura: a review of 272 biopsies. Am J Clin Pathol 1979;72. 48-51.

42. Hirsch JH, Rogers JV, Mack LA. Real time sonography of pleural opacities. AJR 1981;136:297-301.

43. Goralnik CH, O'Connell DM, Yousef SJ, Haaga JR. CT guided cutting needle biopsies in selected chest lesions. AJR 1988;151:903-7.

44. Poe RH, Israel RH, Utell MJ, Hall WJ, Greenblatt DW. Sensitivity, specificity and predictive values of closed pleural biopsy. Arch Intern Med 1984;144:325-8.

45. Boutin C, Rey F. Thoracoscopy in pleural malignant mesothelioma: a prospective study of 188 consecutive patients. Part 1: Diagnosis. Cancer 1993;72:389-93.

46. Loddenkemper R. Thoracoscopy - State of the art. Eur Resp J 1998 11:213-21.

47. Carlens E. Mediastinoscopy: a method for inspection and tissue biopsy in the superior mediastinum. Dis Chest 1959;36:343-52.

48. Geedo A, Van Scil P, Corthouts B, Van Mieghem F, Van Meerbeeck J, Van Marck E. Prospective evaluation of computed tomography and mediastinoscopy in mediastinal lymph node staging. Eur Respir J 1997; $10: 1547-51$

49. Hammoud ZT, Anderson RC, Meyers BF. The current role of mediastinoscopy in the evaluation of thoracic disease. $\mathrm{J}$ Thorac Cardiovasc Surg 1999;118:894-9.

50. Hermanek P, Sobin LH. Union Internationale Contre le Cancer (UICC). TNM classification of malignant tumors. Lung tumors. $4^{\text {th }}$ ed. New York: Spinger-Verlag, 1987;70-3.
51. American Joint Committee for Cancer Staging (AJCC). Manual for staging of cancer. $3^{\text {rd }}$ ed. Philadelphia: Lippincott, 1988;115-21.

52. Denoix PF. Enquete permanent dans les centres anticancereux. Bull Inst Nat Hyg 1946;1:70-5

53. American Joint Committee for Cancer Staging and end Results Reporting (AJCC). Clinical staging system for carcinoma of the lung. Philadelphia: Lippincott, 1973.

54. American Thoracic Society. Clinical staging of primary lung cancer. Am Rev Respir Dis 1993;64:654-9.

55. Bains MS. Surgical treatment of lung cancer. Chest 1991;100:82637.

56. Mountain CF. A new international staging system for lung cancer. Chest 1986;89(Suppl):2255S-335S.

57. Mountain CF. Revisions in the international system for staging lung cancer. Chest 1997;111:1710-7.

58. Naruke T. Significance of lymph nodes metastasis in lung cancer. Semin Thorac Cardiovasc Surg 1993;5:210-8.

59. Mountain CF, Dresler CM. Regional lymph node classification for lung cancer staging. Chest 1997;111:1718-23.

60. Libshitz HI. Computed tomography of mediastinal lymph nodes in lung cancer: is there a state of the art? AJR 1983;141:1081-5.

61. Genereux GP, Howie JL. Normal mediastinal lymph node size and number: CT and anatomic study. AJR 1984;142:1095-100.

62. Kaplan DK. Mediastinal lymph node metastases: is size a valid criterion? Thorax 1992;47:332-3.

63. Fernandez A, Bammann RH, Beyruti R, Junqueira AR, Jatene FB. Avaliação mediastinal no estadiamento do câncer do pulmão. J Pneumol 1998;24:17-22.

64. Fernandez A, Filomeno LTB, Milanez JRC, Jatene FB. Mediastinoscopia: aspectos técnicos e indicações atuais. Rev Hosp Clin Fac Med São Paulo 1992;47:125-7.

65. Fernandez A, Junqueira AR, Beyruti R, Milanez JRC, Cipriano FG, Bammann RH, et al. Importância do diagnóstico histológico na avaliação mediastinal do câncer pulmonar. S Am J Thorac Surg 2000;6: 51-7.

66. Jolly PC, Hutchinson HC, Detterbeck F, Guyton SW, Hoffer B, Anderson RP. Routine computed tomographic scans, selective mediastinoscopy, and other factors in evaluation of lung cancer. J Thoracic Cardiovasc Surg 1991;102:266-71.

67. Ginsberg RJ. Extended cervical mediastinoscopy. A single staging procedure for bronchogenic carcinoma of the upper left lobe. J Thorac Cardiovasc Surg 1987;94:673-5.

68. Gould MK, Lillington GA. Strategy and cost in investigating solitary pulmonary nodules. Thorax 1998;53(Suppl):s32-7.

69. Maddaus M, Ginsberg RJ. Lung cancer: diagnosis and staging. In: Pearson FG. Thoracic surgery, Churchill Livingstone, 1995, 671-90.

70. Sider R, Horejs D. Frequency of extrathoracic metastasis from brochogenic carcinoma in patients with normal sized hilar and mediastinal lymph nodes. AJR 1988;151:893-9.

71. Lowe VJ, Naunheim KS. Positron emission tomography in lung cancer. Ann Thorac Surg 1998;65:1821-9.

72. Weder W, Schmid RA, Brucchaus H, Hillinger S, von Schulthess GK, Steinert HC. Detection of extrathoracic metastases by positron emission tomography in lung cancer. Ann Thorac Surg 1998;66:886-92.

73. Scott WJ, Dewan AD. Use of positron emission tomography to diagnose and stage lung cancer. Clin Pulm Med 1999;6:198-204. 